\title{
Salutogenesis: A new paradigm for pervasive computing in healthcare environments?
}

\author{
C. Dalton, K. McCartney \\ Cork Centre for Architectural Education \\ 9-10, Copley Street, \\ Cork, Ireland
}

\begin{abstract}
Salutogenesis is now accepted as a part of the contemporary model of disease: an individual is not only affected by pathogenic factors in the environment, but those that promote well-being or salutogenesis. Given that "environment" extends to include the built environment, promotion of salutogenesis has become part of the architectural brief for contemporary healthcare facilities, drawing on an increasing evidence-base. Salutogenesis is inextricably linked with the notion of personenvironment "fit'. MyRoom is a proposal for an integrated architectural and pervasive computing model, which enhances psychosocial congruence by using real-time data indicative of the individual's physical status to enable the environment of his/her room (colour, light, temperature) to adapt on an on-going basis in response to bio-signals. This work is part of the PRTLI-IV funded programme NEMBES, investigating the use of embedded technologies in the built environment. Different care contexts require variations in the model, and iterative prototyping investigating use in different contexts will progressively lead to the development of a fully-integrated adaptive salutogenic singleroom prototype.
\end{abstract}

Keywords-salutogenesis; healthcare; intelligent environments; dementia

\section{INTRODUCTION}

The salutogenic model of health was first proposed by Aaron Antonosky in 1979, in an attempt to explain how some individuals maintained good mental health in the face of extremely adverse circumstances [1]. The notion has gained considerable credence not only in the area of medicine, but also in relation to the design of the built environment, where the evidence base to support the notion that a well-designed built environment can positively influence healthcare outcomes has continued to grow over the last 30 years. 'Whilst previously disease was considered only as the product of pathogens and pathogenic factors, there is now considerable emphasis on maintaining good health, and on disease prevention, by maximising salutogenic factors [2]. Among those factors is the built environment [3]. This is obviously of critical importance in the design of healthcare settings, in particular those involving overnight stays for patients.

Antonosky theorises that the concept of salutogenesis is linked to "congruence" or person-environment fit. There are a number of related models of person-environment fit in behavioural psychology, which define congruence in terms of the degree to which an environment provides support for an individual, both functionally and psychosocially [4][5], by providing adequate and appropriate spaces to fulfil both basic and higher needs [6]. Being prevented from fulfilling such needs can lead to stress and illness; physical illness is now recognised as being linked to emotional and mental stress, through the effects of stress on the endocrine system. Lack of congruence can ultimately lead to what Boyden describes, appositely in the case of the older person, as "The Gray Life"[7].

In the case of residential healthcare settings, the ageing demographic in Western society, including in Ireland, implies that a considerable number of additional residential care places is needed imminently to support the demands of a rapidlyageing population [8]. Residential care for elderly people cannot disregard provision for people with cognitive impairment in the form of dementia, most frequently Alzheimer's disease. The question then arises as to how to address the notion of "fit" for such a user: in addition to aspiring to the best standards of design of the built environment, can sensors, actuators and pervasive computing contribute to enhancing the degree of fit between the user and her environment, by ameliorating the effects of both physical and cognitive impairment? Similar considerations apply in critical acute healthcare, where a patient may be immobilised and unable to use controls.

There has long been recognition that prosthetic environments can assist in compensation for loss of both physical and psychosocial functioning [9]. More recent research has involved using new technologies to this end. For example, the potential to extend the use of sensoring and pervasive computing beyond monitoring has been identified [10], including in relation to compensation for cognitive impairment, by utilising real-time sensoring [11]. As yet, little thought has been devoted to promoting well-being though use of information technology and pervasive computing. There is an identified need for such research, specifically for an elderly user-group with dementia, preferably involving inputs from potential end-users[12]. 


\section{MYROOM}

Based on the literature review and interviews with stakeholders, including carers, service-providers designers, and where feasible, with end-users, MyRoom represents an integrated model of ubiquitous computing in a single-room healthcare environment by extending current models to include promotion of salutogenesis. It takes as precedent prototypical intelligent living environments such as AwareHome ${ }^{1}$ and InHaus $2^{\text {ii }}$.

It is envisaged that adaptability is enabled through system responses to real-time bio-sensing of variables such as heart rate, skin resistance and temperature. As systems utilising similar data for monitoring have previously been developed, it is therefore conceivable that therapeutic applications might be conceived as extensions to such systems, where the same sensor data is used to control elements of the building/room management system. The potential use of pervasive computing to assist persons with memory loss and dementia has also been identified [13]. The question is: how best might this be achieved? The MyRoom project is based on the proposition that pervasive computing can be utilised in such a fashion as to maximise person-environment fit, not solely by facilitating functional congruence, but also by actively promoting psychosocial congruence.

\section{METHODOLOGIES}

In addition to literature review, structured interviews, and a design model, research methodologies include the development of an initial prototypical application, (which will ultimately form part of a room management system) enabling activation of a therapeutic visual/multimedia application in response to bio-signals indicative of inappropriate stress. The first application prototype is likely to utilise Shimmer ${ }^{\mathrm{iii}}$ sensors and the Biomobius ${ }^{\text {iv }}$ open research platform, developed by TRIL researchers. In a second stage, a room prototype will be constructed using bespoke sensors and software.

\section{COLOUR AND NATURE IMAGES-THERAPEUTIC BENEFIT}

The literature review has uncovered scant evidence for therapeutic benefits associated with individual colours. This is despite a widely-held belief among the design community and the public that such effects exist. This is not to say that there are no such effects, as few, if any of the methodologies used in empirical colour studies to date are capable of proving or disproving arguments for the efficacy colour as used in the built environment, though some differences in colour preferences relating to both gender and age have been identified. The physical scale of samples in many experiments (e.g. utilising colour-cards, or small images) and the

\footnotetext{
i Georgia Institute of Technology, U.S. http://awarehome.imtc.gatech.edu/

${ }^{\text {ii }}$ Fraunhofer Institute, Duisburg www.inhaus-zemtrum.de

iii http://www.shimmer-research.com/

${ }^{\text {iv }}$ http://www.biomobius.org/
}

conclusions drawn in a number of cases provide results which are unconvincing to the designer of the built environment, or of little or no benefit in practice. Over-zealous and narrow interpretation of the results of such research can hinder rather than facilitate good design. Nonetheless, there is still an amount of credible literature regarding use of colour in the built environment [14], and the benefits of using specific colours to maximise human functioning in specific settings [15]. As the MyRoom proposal incorporates response to an individual's biosignals, and indication of favourable response (i.e. restoration of appropriate stress levels), room colour can readily be adjusted to those which maximise any individual occupant's well-being, also allowing for future adjustment in the light of any new research evidence demonstrating specific therapeutic benefit which may emerge.

There is however convincing research to support the notion that views of natural scenes reduce stress levels, of which the work of Roger Ulrich is best-known. [16]. This points the way to development of a multimedia application utilising such images. Digitisation readily facilitates personalisation of images for an individual, and any such application might therefore also include elements of reminiscing.

\section{PROTOTYPING}

Because of ethical and practical considerations, initial prototyping will not involve dementia patients. Miniaturisation of sensors, and the development of unobtrusive wearable sensors will in time facilitate research/ prototyping involving end-users in live healthcare environments [17] [18]. Because of the particular challenges and restrictions in relation to vulnerable groups, as epitomised by persons with dementia, it is likely that applications suitable for use in acute hospital environments will the first to be developed, outside the scope of the current doctoral research programme. Such applications might then be further modified or augmented to render them more suitable for use in a long-stay environment, taking cognisance of the specific needs of persons with dementia. (PWD). There are specific issues in relation to wearable sensors for this user-group, as there is not only a likelihood of removal or damage to the sensor, but it may in itself constitute further stress as an alien/unfamiliar object. The possibility of using embedded room sensors must therefore also be considered. This approach has been used in research into behavioural patterns of elderly people [19].

In any scenario, machine-learning will form an essential element of the model for all but the most basic prototype, if the room environment is to be rendered truly adaptable to an individual user. The development of the prototype, both with respect to individual applications and ultimately an integrated room model, will of necessity be an iterative process, which mirrors the design process for architectural spaces and buildings. Prototypical applications, such as the multimedia application described, will first be individually tested for functionality, and ultimately integrated into a room prototype which will necessitate further testing for integrated 
functioning, including testing of custom middleware required to achieve this.

A comprehensive room prototype will integrate multiple applications for environmental control, including lighting, colour, temperature and sound, and permit addition of further applications as the knowledgebase regarding human/ builtenvironment interaction broadens. Because of humanistic and ethical considerations, it should allow also for direct personal control of applications by the user, via simplified purposedesigned interfaces where appropriate: ability to control/ modify the environment is an important factor in achieving optimal person-environment fit, though machine -learning is likely to be of greater significance in a situation where the user has lost the physical and/or cognitive ability to operate manual or even graphic interfaces.

\section{SCOPE OF CURRENT RESEARCH}

The doctoral research will define a physical model for the room and its technological infrastructure. It will also examine adaptability options facilitated by pervasive computing. The proposal is set in the context of a new model of residential care located between the current extremes of "ageing-in-place" in one's own residence, and the traditional institutional care setting. While such models currently exist [20], and have been tentatively accepted in modified form by some public healthcare providers ${ }^{\mathrm{v}}$, closer scrutiny is required in order to determine the extent to which such care models, as physically realised, match the intentions or expectations of the original concept. The significance of the physical context cannot be underestimated, given its influence on person/ environment fit. In the light of the current reality regarding existing institutional care, therapeutic applications as described may have more immediate potential in the context of retrofit, in order to immediately ameliorate or improve existing care settings which do not measure up to new or developing standards. There is also potential wider application in the built environment generally in any single-room setting.

\section{DISCUSSION}

There has been some exploration of utilising feedback generated by bio-sensors in the area of performance art, including modification of live performance, using Eyesweb open-source software. Pervasive computing may offer the means by which architecture and the built environment is similarly enabled to adapt to the user in an almost "intuitive" fashion.

It is hoped that the qualitative description of the room model will provide the springboard for discussion of a pervasive computing model for a prototypical room.

\section{REFERENCES}

[1] A. Antonosky, "The salutogenic perspective: Toward a new view of health and illness", Advances institute for the Advancement of Health, vol. 4 issue 1, pp. 47-55, 1987.

[2] A. Dilani, "Psychosocially supportive design: a salutogenic approach to the design of the physical environment", World Health Design, pp. 4755, July 2008.

[3] B. Lawson, M. Phiri, "The Architectural Healthcare Environment and its Effects on Patient Outcomes", TSO, 2003.

[4] S. Kaplan, "A model of person-environment compatibility", Environment \& Behavior.

[5] E. Kahana, "A congruence model of person-environment interaction," in T.O. Byerts, M.P. Lawton, J. Newcomber, J. (Eds.) Theory development in environment and aging, Washington, DC: Gerontological Society, 1975.

[6] A. H. Maslow, "A Theory of Human Motivation" Psychological Review, issue 50, pp. 370-396, 1943.

[7] S. Boyden, "Biological Determinants of optimal health", in D. J. M. Vortster, (Ed) The human biology of environmental change: conference proceedings., Blantyre, Malawi, 1971.

[8] Richard Layte (ed.) et al "Projecting The Impact Of Demographic Change On The Demand For And Delivery Of Health Care In Ireland" Dublin, Ireland, ESRI, Research Series 13, 2009.

[9] L. E. McClannahan, "Therapeutic and Prosthetic Living Environments for Nursing Home Residents", The Gerontologist vol. 13, issue 4, 1973, pp. 424-429.

[10] A. Lymberis. "Smart Wearables for Remote Health Monitoring, from Prevention to Rehabilitation: Current R\&D, Future Challenges." In 4th International IEEE Engineering in Medicine and Biology Society Special Topic Conference on Information Technology Applications in Biomedicine, Birmingham, UK, 2003, pp. 272-275.

[11] L. Atallah, G-Z Yang, "The use of pervasive sensing for behaviour profiling a survey", Pervasive and mobile computing, vol. 5, issue 5, October 2009, pp. 447-464.

[12] A. J. Sixsmith, G. Gibson, R. D. Orpwood, J. Torrington, "Developing a technology wish-list to enhance the quality of life of people with dementia". Gerontechnology, vol. 6, issue 1, 2007, pp. 2-19.

[13] L. Atallah, G-Z Yang, "The use of pervasive sensing for behaviour profiling a survey", Pervasive and mobile computing, vol. 5, issue 5, October 2009, pp. 447-464.

[14] Mahnke \& Mahnke "Colour and light in manmade environments"

[15] F. Birren, "Colour Psychology and Colour Therapy" New York, McGraw-Hill .1950.

[16] R. S. Ulrich, "Natural versus urban scenes - some psychophysiological effects". Environment and Behavior, issue 13, 1981, pp. 523-556.

[17] S. Bamberg, A. Y. Benbasat, D. M. Scarborough, D. E. Krebs, and J. A. Paradiso. "Gait Analysis using a Shoe-Integrated Wireless Sensor System." In IEEE Transactions on Information Technology in Biomedicine, vol. 12, no. 4, pp. 413-423, 2008.

[18] A. Lymberis. "Smart Wearables for Remote Health Monitoring, from Prevention to Rehabilitation: Current R\&D, Future Challenges." In 4th International IEEE Engineering in Medicine and Biology Society Special Topic Conference on Information Technology Applications in Biomedicine, Birmingham, UK, 2003, pp. 272-275.

[19] L. Atallah, G-Z Yang, "The use of pervasive sensing for behaviour profiling _ a survey", Pervasive and mobile computing, vol. 5, issue 5, October 2009, pp. 447-464.

[20] A. McLean "New Approaches to Nursing Home/ Dementia Care in the US", ICSG, NUI Galway, unpublished.

\footnotetext{
${ }^{\vee}$ e.g. HSE's Teaghlach initiative
} 
$\longrightarrow$ 